\title{
LA CREATIVITÀ DELLE FOTOGRAFIE ON-LINE ALLA LUCE DELLA NUOVA DIRETTIVA 790/2019
}

Bianca Trerè

Collaboratrice della cattedra di Copyright Law Scuola di Giurisprudenza Dip. Scienze Giuridiche Alma Mater Studiorum, Università di Bologna 
Lo sviluppo del rapporto tra copyright e nuove tecnologie in tema di circolazione e diffusione digitale delle opere è direttamente proporzionale a due variabili costanti della società odierna: dinamismo e accelerazione dei tempi.

Il complesso normativo che regola la materia (essenzialmente le direttive n. 2019/790 sul diritto d'autore e sui diritti connessi nel mercato unico digitale e n. 2001/29 sull'armonizzazione di taluni aspetti del diritto d'autore e dei diritti connessi nella società dell'informazione) presenta confini labili e mutevoli, al punto che le autorità giudiziarie statali e comunitarie sono chiamate non solo a dirimere controversie, ma anche a fornirne l'interpretazione: una valutazione normativa aggravata dalla circostanza per cui i giudici sono chiamati a pronunciarsi non solo sugli elementi giuridici quanto nell'ambito di una vera fase di "politica del diritto". In un contesto del genere il vero elemento innovativo di cui deve tenere conto l'interprete è rappresentato da fattori umani, quali la modifica della percezione del tempo e della realtà che creano una potenziale frattura fra ambito normativo e sociale.

Fra gli interventi giurisprudenziali in materia, la pronuncia di Corte Giust. UE, 7 agosto 2018, C-161/17, "Land Nordrhein- Westfalen C. Dirk Renckhoff", introduce stimoli che, ad avviso di chi scrive, possono riflettersi nel delineare una linea interpretativa della nuova direttiva sopra citata. La vicenda giurisprudenziale surrichiamata si risolve in un'applicazione automatica e letterale della norma vigente all'epoca dei fatti: tuttavia, non sono pochi gli aspetti farraginosi. I diritti che entrano in gioco diventano oggetto di una complessa operazione di bilanciamento, che sebbene culmini, come si è detto, in un intervento giurisprudenziale normativamente coerente, apre un dibattitto acceso su aspetti afferenti la direttiva InfoSoc.

Brevemente, la questione pregiudiziale proposta alla Corte, aveva preso le mosse da una controversia proposta innanzi alla Corte federale di giustizia tedesca concernente l'uso, a titolo illustrativo ma non autorizzato, di una fotografia della città di Cordova scattata dal fotografo Dirk Renckhoff, da parte di una studentessa della Gesamtschule di Waltrop; quest'ultima, nello svolgere un progetto scolastico di laboratorio linguistico, aveva infatti dapprima scaricato e poi pubblicato sul sito Internet del proprio istituto la fotografia oggetto del procedimento, già disponibile su un sito Internet dedicato ai viaggi, sul quale la stessa era stata inizialmente pubblicata con il consenso del suo autore. Renckhoff, asserendo una lesione dei propri interessi e ribadendo di aver autorizzato I'uso unicamente sul primo sito, affermava che la messa in rete della fotografia sul secondo costituisse una violazione del proprio diritto d'autore'

La questione principale di cui la Corte si occupa in via pregiudiziale è quella di delineare la nozione di comunicazione al pubblico, stabilendo se il caso di specie potesse e dovesse costituire ipotesi di sottoposizione ad un pubblico nuovo (e quindi diverso) rispetto a quello originario e autorizzato dall'autore dell'opera. La dottrina ha analizzato in profondità le pronunce della Corte riconoscendo in più occasioni i tentativi di dare uniformità alla lettura della materia. Invero l'insieme dei criteri complementari addottati nelle diverse decisioni di natura oggettiva o soggettiva, di volta in volta indipendenti o messi in relazione fra loro, fanno emergere un'unica linea guida: I'approccio analitico tende ad individuare e regolare il caso concreto, rendendo in questo modo la decisione

\footnotetext{
${ }^{1}$ Cfr. DEL PLATO, Caso Land Nordrhein-Westfalen: la vittoria del copyright sulla "sfrenata" condivisione dei contenuti in rete, in Media Laws, 2019
} 
più equa ma impedendo la formazione di una regola unitaria che possa creare un vero e utile precedente ${ }^{2}$.

In quest'ordine, imprescindibile elemento da prendere in considerazione ai fini della risoluzione della controversia non può che riguardare la tecnica di comunicazione, ovvero il mezzo di Internet. Come rilevato nelle conclusioni dell'avvocato generale, "la messa a disposizione costituisce nel mondo digitale l'equivalente dell'atto di comunicazione nel mondo analogico" ${ }^{3}$ ed è proprio da questo assunto che potrebbe essere interessante partire: si è di fronte ad un vaglio che per quanto tecnico non può prescindere dal contesto in cui il contenuto deve attecchire. Internet non è più uno strumento comunicativo, ma lo strumento. La rivoluzione che si è verificata è troppo recente ed ancora in fase di burrascosa evoluzione: lo storico non può ancora studiarne il carattere o descriverne gli effetti ma certamente il nostro mondo è radicalmente cambiato e le persone che lo abitano insieme a lui. "Non è solo una rivoluzione tecnologica fatta di nuovi oggetti, ma il risultato di un'insurrezione mentale." ${ }^{4}$ Su quest'onda "rivoluzionaria", ogni contenuto diventa immensamente più fruibile ed accessibile e contemporaneamente instabile, mobile, "liquido" ${ }^{5}$. La stessa variazione si ha in termini quantitativi: non solo un incremento esponenziale del numero di contenuti, ma anche di quello dei fruitori in grado di accedere a quello stesso contenuto.
Ne deriva che, da un lato, vi è un soggetto che scientemente decide di pubblicare in rete la propria opera creativa e, dall'altro, una massa indistinta e indeterminata di soggetti messi nella condizione di potervi accedere. La Corte ha infatti dichiarato che "la comunicazione su un sito internet, senza limitazioni di accesso, è diretta a tutti gli utilizzatori potenziali, denominati internauti, di tale sito".6 $\dot{E}$ subito il caso di rilevare che un rapporto del genere difficilmente può concepirsi neIl'ambito di un rapporto classico di diritto privato a partire dalla ripartizione della responsabilità: ad un normale utente di Internet non si può richiedere una diligenza superiore rispetto al titolare del diritto d'autore. Questo discrimen di competenza risulta ulteriormente accentuato nell'ambito di rapporti, come nel caso di specie, in cui una delle parti sia evidentemente debole in quanto studentessa7. Partendo dal presupposto che risulta impensabile ad oggi non avvalersi di Internet per eseguire anche la più banale delle ricerche è lecito presumere, proprio per la debolezza della parte, che l'autore non si opponga all'uso limitato di immagini da lui stesso pubblicato laddove esse siano utilizzate a scopo didattico. Ed invero, l'ambito di utilizzo del contenuto (un progetto promosso dalla scuola e svolto dagli studenti), rende evidente I'assenza di fine lucro alla base dell'utilizzo della creazione ed è proprio da questa "assenza di clienti" che si desume una giustificabilità in capo all'alunna. Si ritiene infatti che da questa

\footnotetext{
${ }^{2}$ Cfr. SPEDICATO, II diritto di comunicazione e messa a disposizione del pubblico, in AIDA, 2017,264 ss.

${ }^{3}$ Cit. Corte Giust. UE, 7 agosto 2018, C-161/17, Conclusioni dell'avvocato generale, § 4.

${ }^{4}$ Cit. BARICCO, The Game, Einaudi, 2018.

${ }^{5}$ II concetto di "società liquida" è stato elaborato dal sociologo polacco BAUMAN secondo il quale la modernità è la convinzione che il cambiamento è l'unica cosa permanente e che l'incertezza è I'unica certezza. Moderinità liquida, Edizioni Laterza, 1999.

${ }^{6}$ Cit. Corte Giust. UE, 7 agosto 2018, C-161/17, Conclusioni, § 93. Cfr. Corte Giust. UE, 29 novembre 2017, C-265/16, §§ 46,47 (VCAST) e Corte Giust. UE, 13 febbraio 2014, C-466/12, § 22 (Svensson e a.)

${ }^{7}$ Cfr. Corte Giust. UE, 7 agosto 2018, C-161/17, Conclusioni, § 78.
} 
condizione discenda una generale attitudine del soggetto a non agire con piena cognizione delle conseguenze ${ }^{8}$.

Già l'art. 3 lett. a) della direttiva n. 2001/29 disciplinava la c.d. "eccezione didattica" conferendo agli Stati la facoltà di disporre eccezioni e limitazioni ai diritti del titolare "allorché I'utilizzo ha esclusivamente finalità illustrativa per uso didattico o di ricerca scientifica, sempreché, salvo in caso di impossibilità si indichi la fonte, compreso il nome dell'autore, nei limiti di quanto giustificato dallo scopo non commerciale perseguito;". Orbene, è palese il contesto in cui l'attribuita nuova comunicazione al pubblico veniva effettuata: un progetto scolastico in cui la studentessa inseriva una fotografia per rendere più espliciti i contenuti. Attività che di per sé non porta la stessa a ritenere di compromettere nessuno, né tantomeno di ledere diritti altrui, al punto che nel sito scolastico vengono indicati i riferimenti da cui la fotografia viene presa e trasposta. La pronuncia emessa dalla Corte segue l'impostazione sottesa all'impianto normativo del 2001 volto a consentire una tutela piena ed effettiva del diritto d'autore. Per quanto siano previste eccezioni e limitazioni al diritto, esse si manifestano come ipotesi facoltative, e comunque deboli perché dai confini troppo incerti. Infatti, così come rilevato dall'avvocato generale nelle sue conclusioni, sebbene la norma appaia "chiara nella sua incertezza", non si può fare a meno di pensare che in sede di bilanciamento con altri diritti riconosciuti come fondamentali dalle stesse fonti comunitarie (diritto all'istruzione), la protezione autoriale non possa rischiare di incidere eccessivamente sulla loro efficacia ed attuazione. Come premesso dunque, il tipo di eccezione proposta dal legislatore del 2001 risulta caratterizzata da un profilo di facoltatività riconosciuta in capo a ciascuno stato di intervenire in misura più o meno incisiva nel determinare la limitazione al diritto del titolare dell'opera. Non viene quindi ravvisata in sede di giudizio dalla Corte l'ipotesi di eccezione didattica in quanto si ritiene dover essere preminente la tutela del diritto d'autore del sig. Renckhoff; la decisione genera non poche perplessità e rende difficile comprendere quale dei due opposti orientamenti sia maggiormente condivisibile.

Alla luce dell'emanazione della nuova direttiva n. 2019/790 potrebbe ritenersi utile, ai fini di un'interpretazione più certa fin dai suoi primi esordi applicativi, una ri-valutazione prodromica del caso in oggetto: com'è noto, lo stesso art. 5 della direttiva (rubricato per l'appunto "utilizzo di opere e di altri materiali in attività didattiche digitali e transfrontaliere", in richiamo alla fonte normativa precedente), prevede la figura dell'eccezione didattica. Occorre premettere, che la direttiva in commento si inserisce in un quadro ben preciso, così come rilevato dai consideranda relativi ad essa: scopo ultimo è quello di un'ulteriore armonizzazione delle legislazioni degli Stati membri onde evitare una frammentazione del mercato interno. Attraverso il quadro giuridico si mira a stimolare l'innovazione, la creatività, gli investimenti e la produzione dei contenuti anche in ambiente digitale: sono infatti i rapidi sviluppi tecnologici a minare la tempestività della legislazione rispetto alle esigenze avvertite. Sebbene la prerogativa ri-

\footnotetext{
${ }^{8}$ Con riguardo al caso concreto, sebbene non lo dichiari in maniera esplicita, anche nelle decisioni in oggetto la Corte segue il precedente di Corte Giust. UE, 8 settembre 2016, C-160/15 (GS Media), in base al quale nel caso in cui si agisca senza fini lucrativi, debba essere dimostrata la consapevolezza dell'illegittimità della pubblicazione dell'opera, per cui occorre tenere conto di tutte le circostanze e degli elementi specifici di ciascun caso.
} 
manga quella di tutelare in modo permeante la condizione dell'autore, nuovi spiragli si aprono con riferimento proprio all'ipotesi della figura deIl'eccezione didattica, sancendo il legislatore per essa il carattere dell'obbligatorietà: impostazione che impone di riflettere su quali potrebbero essere stati gli sviluppi della vicenda "Renckhoff" se giudicati oggi. In particolare si ravvisa come le tecnologie digitali consentano nuovi usi (anche non esplicitamente contemplati) nei settori della ricerca, dell'innovazione e dell'istruzione al punto da ritenere il precedente carattere facoltativo dell'eccezione didattica lesivo del buon funzionamento del mercato interno. Entrando più nel merito, la direttiva contrappone all'obbligatorietà dell'eccezione didattica due condizioni: I'ambiente in cui l'attività in questione deve essere esercitata, sia esso fisico o elettronico e caratterizzato da accesso limitato, e l'indicazione nella fonte del nome dell'autore del contenuto utilizzato, laddove possibile. La presunzione di buona fede dell'alunna e del docente possono essere desunte dalla dicitura in calce alla fotografia del sito di viaggi autorizzato a pubblicare il contenuto e in cui era possibile attingere. Manca tuttavia un limite di accesso al blog scolastico tale da impedirne una visione generalizzata e ad libitum: I'eccezione o limitazione dovrebbe riguardare sia I'utilizzo di opere o altri materiali in classe o in altre sedi mediante strumenti digitali, sia I'utilizzo a distanza all'interno di un ambiente informatico sicuro ${ }^{9}$. Questo contrasto con i requisiti summenzionati ai fini della configurazione dell'eccezione didattica confligge tuttavia con un elemento di fatto molto forte ovvero la pubblicazione dell'opera oggetto in un sito internet privo di qualsiasi misura restrittiva, non solo ai fini di visione, ma anche di copiatura. Nessuna elusione di sistema viene infatti attribuita alla scuola, al docente, né tantomeno alla studentessa e, ad onor del vero, il contenuto risultava già liberamente accessibile e fruibile sul sito di viaggi autorizzato.

Per una corretta analisi, da ultimo, si deve tener conto della difficoltà nel determinare lo standard creativo necessario per l'attribuzione della tutela d'autore alle immagini realizzate tramite processo fotografico. La nozione stessa di creatività non si presta facilmente ad una definizione normativa: l'interprete non può prescindere da un'indagine concreta e fattuale dell'opera creata e dei suoi precedenti. In questo senso, ancorare il concetto di creatività ai canoni di certezza richiesti dal diritto risulta difficile e talvolta addirittura incoerente data la sua natura meta-giuridica. Ne deriva che pretendere di concepire la creatività in un'ottica di assolutezza rischierebbe di esasperare il contenuto del diritto d'autore al punto da paralizzare il processo creativo stesso ${ }^{10}$. La ricerca del minimum creativo che rende l'opera tutelabile potrebbe però portare a riconoscere l'esistenza di una creatività di serie a) e una di serie b). Stabilita dunque l'esigenza logico-giuridica nella valutazione di merito creativo e volendo ora riallacciarsi alla vicenda Renckhoff, è necessario premettere che l'evanes-

\footnotetext{
${ }^{9}$ Sul punto si veda il considerando n.22 della direttiva n. 790/2019, laddove si afferma che "per ambienti informatici sicuri si dovrebbero intendere gli ambienti digitali di insegnamento e di apprendimento ai quali possono accedere unicamente il personale docente dell'istituto di istruzione e gli alunni mediante apposita procedura di autenticazione che comprenda una password".

10 Sul punto MUSSO, Diritto d'autore sulle opere dell'ingegno letterarie e artistiche, Zanichelli, 2008 , 24 s; laddove si ritiene non condivisibile "un'indiscriminata protezione in favore di ogni spicciolo creativo, dovendosi sempre attribuire la qualifica di opera di ingegno sia in relazione al bilanciamento di meritevolezza rispetto ai diritti dei terzi, sia in rapporto alla necessaria estrinsecazione della creatività nell'opera stessa."
} 
cenza della valutazione di creatività è ulteriormente accentuata nell'ambito delle opere fotografiche in quanto frutto di un procedimento meccanico. A questo "automatismo" viene però correlato il riconoscimento alla fotografia come veicolo di informazione il cui pregio e valore economico non dipendono solo dal carattere creativo "ma anche dal carattere di documento di un accadimento o di una realtà difficilmente accessibile".11 La peculiarità della vicenda Renckhoff risiede certamente anche in questo: può considerarsi la fotografia oggetto della questione pregiudiziale presentata alla Corte, dotata di carattere creativo ${ }^{12}$ È plausibile pensare che nella società dell'informazione uno scatto come quello in esame non finisca con l'essere uno fra i tanti? Ed è proprio fra quei "tanti" che il legislatore oggi deve muoversi, quei grandi numeri frutto di un effetto di rete imperante ma anche spudorato. Tuttavia il nucleo essenziale del processo creativo, a prescindere dalla sua estrinsecazione finale, rimane puramente umano ed è proprio attraverso l'osservazione reciproca che si genera: creatività crea creatività.

Ad analoga considerazione si potrebbe infine giungere con riguardo alla disciplina dei diritti connessi al diritto d'autore ${ }^{13}$. Invero, nel testo originario della legge d'autore non venivano ricomprese le opere fotografiche nella categoria di cui all'art. 2: per sopperire a tale mancanza nonché per superare l'ostacolo della determinazione del carattere creativo (particolarmente stringente, come si è detto, nel processo fotografico), il legislatore del '79 andò a configurare un doppio livello di protezione, inserendo anzitutto le fotografie neII'elenco di cui all'art. 2 I. aut. (al n. 7), ma stabilendo che solo per quelle dotate di elemento creativo sarebbe stato possibile accedere alla tutela del diritto d'autore ${ }^{14}$, mentre per tutte quelle fotografie c.d. "semplici", descritte come "immagini di persone o di aspetti, elementi o fatti della vita naturale e sociale, ottenute col processo fotografico o con processo analogo, comprese le riproduzioni di opere dell'arte figurativa e i fotogrammi delle pellicole cinematografiche", viene accordata la tutela meno forte del diritto connesso di cui all'art. 87 I. aut. $^{15}$ In considerazione dei fatti in oggetto, malgrado la parte convenuta non abbia sollevato in sua difesa l'eccezione di creatività, si potrebbe

${ }^{11}$ Cfr. AUTERI, Diritto industriale, Giappichelli, 2016, 595, laddove si affronta la disciplina del diritto d'autore delle opere fotografiche, nonché il problema di determinare lo standard di creatività: tale compito, affidato alla giurisprudenza non è semplice perché "un minimo di creatività è presente in ogni fotografia."

${ }^{12}$ Sul punto si vedano i requisiti individuati in Corte Giust. UE, $1^{\circ}$ dicembre 2011, C-145/10 (Painer): in forza di tale sentenza, un ritratto fotografico può essere protetto dal diritto d'autore, "alla condizione che costituisca una creazione intellettuale dell'autore che ne riflette la personalità e si manifesta attraverso le libere scelte creative di quest'ultimo nella realizzazione di tale ritratto". Nella giurisprudenza italiana si segnala: Trib. Di Bari sez. spec. propr. industr. ed intell., 22.07.2010, n. 2606.

13 I diritti connessi integrano una categoria relativamente aperta e sempre più espansiva, con la finalità di limitare la tutela del diritto d'autore unicamente alle opere che estrinsecano un sufficiente grado di creatività. Se ne deduce a contrario che i diritti connessi hanno ad oggetto opere prive di originalità, "delimitando verso il basso" quelle invece protette dal diritto d'autore. ARE, L'oggetto, 491; Cfr. MUSSO, Diritto d'autore sulle opere dell'ingegno letterarie e artistiche, Zanichelli, 2008, 299.

${ }^{14}$ Con riguardo alla tutela diversificata nelle opere fotografiche in base alla determinazione del livello creativo, si rinvengono numerose pronunce giurisprudenziali; volendone indicare alcune: Tribunale Roma sez. IX, 26/06/2017, n.13045; meno recenti, ma comunque incisive: Cass. civ. sez. I, 04/07/92, n. 8186 e Cass. civ. sez. I, 21/06/2000, n. 8425.

15 In base ad art. 87, 2 c., I. aut., nessuna tutela invece è accordata alle "fotografie di scritti, documenti, carte di affari, oggetti materiali, disegni tecnici e prodotti simili." 
dubitare non solo dell'applicazione della tutela più forte del diritto d'autore, bensì anche di quella più "blanda" del diritto connesso, per meritevolezza sul piano del rilevante investimento finanziario o personale da parte del fotografo per un sì fatto scatto. Si potrebbe infatti dubitare che, a tal proposito, I'implicita ratio sottesa al riconoscimento del diritto connesso sia, nel caso in esame, rinvenibile: sebbene solo l'art. 102 bis I. aut. (in riferimento alle banche dati) richiami esplicitamente I'impiego di mezzi finanziari, di tempo e di lavoro nella costituzione dell'opera, è pacifico che lo sweat of the brow debba sussistere anche per la tutela degli oggetti dei diritti connessi ${ }^{16}$.

$\mathrm{Ci}$ si può chiedere, in conclusione, come sarebbe stata decisa la fattispecie in esame all'interno dell'odierno quadro normativo. Il legislatore della direttiva n. 790/2019, nell'ottica di un'operazione di bilanciamento che vede contrapposti la tutela autoriale e gli interessi, nonché i diritti, dei terzi, introduce ex novo la disciplina riguardante la scadenza della durata di protezione di un'opera delle arti visive, sancendo (all'art. 14) che il materiale derivante da un atto di riproduzione di tale opera non sia soggetto al diritto d'autore o a diritti connessi, a meno che il materiale risultante sia originale nel senso che costituisca una creazione intellettuale propria dell'autore. Appare prima facie evidente l'intento del legislatore, consapevole non solo della necessità di invertire il c.d. effetto boomerang dell'overprotection ${ }^{17}$, ma anche di contestualizzare il diritto d'autore e i suoi diritti connessi nel nuovo ambiente digitale: se è vero da un lato che la scadenza dei diritti delle opere visive favorisce l'accesso alla cultura e la sua promozione (soddisfacendo quindi l'esigenza di tutela dei diritti in capo ai terzi), è altrettanto inopinabile che l'attuale società dell'informazione, che si avvale principalmente di strumenti digitali per la circolazione dei materiali visivi, rischia di vanificare I'intento della disciplina della durata del diritto d'autore sulle opere protette ${ }^{18}$. Diretta conseguenza di quanto fin d'ora argomentato è dunque che alcune riproduzioni di opere delle arti visive divenute di dominio pubblico non dovrebbero essere protette dal diritto d'autore o da altri diritti connessi ed è pertanto auspicabile un recepimento, in tal senso conforme, anche dalle stesse autorità giurisdizionali al fine di garantire effettiva efficacia alle disposizioni in oggetto.

\footnotetext{
${ }^{16}$ Per concretizzare il concetto di sweat of the brow nelle opere fotografiche v. la recentissima pronuncia del Tribunale di Roma , sez. XVII , 12/09/2019 , n. 14758, secondo cui: “La fotografia quale opera d'arte, e come tale tutelabile ex artt. 2 e 87 ss. I.d.a. , presuppone una lunga e accurata scelta, da parte del fotografo, del luogo, del soggetto, dei colori, dell'angolazione, dell'illuminazione, e si concretizza in uno scatto unico, irripetibile nel quale l'autore sintetizza la sua visione del soggetto. II fotografo deve, quindi, avere in mente un obiettivo pittorico e creativo di valore artistico ed innovativo che tende a realizzare in una rappresentazione che non è grafico-pittorica bensì fotografica. In sostanza i presupposti per riconoscere ad una fotografia valore di opera d'arte sono i medesimi che devono essere ascritti ad un quadro. La fotografia deve essere l'espressione di un progetto artistico, di uno stile, di un momento creativo. Di conseguenza, non può essere riconosciuta tutela, quale opera d'arte fotografica, a uno scatto compiuto in occasione di un convegno e nell'adempimento di un contratto di impiego, che può qualificarsi come una testimonianza, a mo' di cronaca, di una situazione di fatto."

17 Cfr. MASKUS e REICHMAN, The globalization of private knowledge goods and the privatization of global public goods, in Propriété, a cura di GENDREAU, 377, con ulteriori riferimenti.

${ }^{18}$ Cfr. Considerando n. 53, dir. UE n. 790/2019, laddove inoltre si afferma che le differenze tra le legislazioni nazionali in materia di diritto d'autore che disciplinano la protezione di tali riproduzioni causano incertezza giuridica e incidono sulla diffusione transfrontaliera delle opere delle arti visive di dominio pubblico.
} 
